\title{
Primary Cardiac Lymphoma: A Case Report
}

\author{
Firas Ajam $^{\mathrm{a}, \mathrm{d}}$, Priyanka Batchu ${ }^{\mathrm{a}}$, Kyle Wiseman ${ }^{\mathrm{b}}$, Dhairya Gor $^{\mathrm{b}}$, Elisabeth Mason ${ }^{\mathrm{b}}$, Abbas Alshami $^{\mathrm{b}}$, \\ Steven Douedi ${ }^{b}$, Aditya Mehra ${ }^{\mathrm{a}}$, Ravneet Bajwa ${ }^{\mathrm{c}}$, Michael Levittc ${ }^{\mathrm{c}}$, David Greenberg ${ }^{\mathrm{c}}$, Muhammad Raza ${ }^{\mathrm{a}}$
}

\begin{abstract}
Cardiac lymphoma is a rare neoplasm involving heart, pericardium or both, usually seen in immunocompromised patients. We report a 61-year-old male presenting with worsening shortness of breath and 20-pound weight loss. Investigations showed right-sided tumor with interatrial septal wall perforation and left atrial expansion. The diagnosis was confirmed with mediastinal mass biopsy. After receiving the appropriate treatment, there was a steady improvement clinically and on the transesophageal echocardiography.
\end{abstract}

Keywords: Cardiac lymphoma; Arrhythmias; Chemotherapy; Cardiac tumors

\section{Introduction}

Primary cardiac lymphoma is a rare disease, mostly seen in immunocompromised patients. Wide range of symptoms upon presentation includes shortness of breath, chest pain, arrhythmias, or syncope. Given the nonspecific nature of symptoms, diagnosis might be challenging. Diffuse large cell lymphoma is the most common type. Different chemotherapy regimens have been used. Herein, we are describing the clinical course of a patient found to have diffuse large B-cell lymphoma (DLBCL) involving the right side of the heart.

\section{Case Report}

\section{Investigations}

This is a 61-year-old non-smoker male with a past medical his-

Manuscript submitted August 28, 2021, accepted September 16, 2021

Published online November 5, 2021

${ }^{a}$ Cardiology Department, Jersey Shore University Medical Center, Neptune, NJ, USA

${ }^{b}$ Internal Medicine Department, Jersey Shore University Medical Center, Neptune, NJ, USA

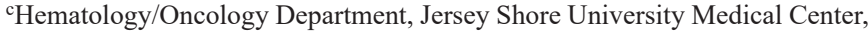
Neptune, NJ, USA

${ }^{\mathrm{d} C}$ Corresponding Author: Firas Ajam, Department of Cardiology, Jersey Shore University Medical Center, 1945 Route 33, Neptune, NJ 07753, USA.

Email: Firas.Ajam@hmhn.org

doi: https://doi.org/10.14740/jmc3773 tory significant for hypertension, hyperlipidemia, obesity, body mass index (BMI) of 30, and sleep apnea, who presented with exertional shortness of breath and dry cough for 3 months, associated with unintentional 20-pound weight loss, low appetite and frequent night sweats. He denied any chest pain, palpitations, fever, abdominal pain or nausea. Physical examination was only significant for bilateral rales and wheezes, bilateral lower extremities pitting edema, and an irregular bradycardic rate and rhythm, and normal heart sounds with no murmur, otherwise unremarkable.

In the emergency room (ER), he was found to be afebrile, blood pressure (BP) 161/69 $\mathrm{mm} \mathrm{Hg}$, heart rate (HR) 50 beats per minute (bpm), respiration rate (RR) 22 and oxygen saturation $\left(\mathrm{SPO}_{2}\right) 98 \%$ on room air. Electrocardiogram (EKG) showed atrial flutter 4:1 block (Fig. 1). Labs results were: white blood cells (WBCs) $12.0 \times 10^{3} / \mu \mathrm{L}$, hemoglobin $(\mathrm{Hg})$ $11.4 \mathrm{~g} / \mathrm{dL}$, creatinine $(\mathrm{Cr}) 2.33 \mathrm{mg} / \mathrm{dL}$, B-type natriuretic peptide (BNP) $236 \mathrm{pg} / \mathrm{dL}$, TPI $0.01 \mathrm{ng} / \mathrm{mL}$, D-dimer 1,156 ng/ $\mathrm{mL}$, international normalized ratio (INR) $2.14, \mathrm{Mg} 1.4 \mathrm{mg} /$ $\mathrm{dL}$, Na $129 \mathrm{mEq} / \mathrm{L}, \mathrm{K} 3.8 \mathrm{mEq} / \mathrm{L}$, normal B-HcG, normal $\alpha$-fetoprotein (AFP), SPEP no monoclonal protein identified, negative human immunodeficiency virus (HIV), negative hepatitis panel, uric acid $8.8 \mathrm{mg} / \mathrm{dL}$ (normal range: $4-8 \mathrm{mg} / \mathrm{dL}$ ), and lactate dehydrogenase (LDH) $381 \mathrm{U} / \mathrm{L}$ (normal range: 80 - $200 \mathrm{U} / \mathrm{L}$ ), portable chest X-ray showed large bilateral pleural effusions with multiple consolidations. Doppler ultrasound and $\mathrm{V} / \mathrm{Q}$ scan were negative.

\section{Diagnosis}

Patient was thus admitted to the hospital and was started on low-intensity heparin drip. Transesophageal echocardiogram (TEE) was performed and showed: 1) interatrial septal wall thickening; 2) tricuspid valve mass; and 3) interatrial septal wall perforation and tumor expansion to left atrium (Fig. 2). Non-contrast computed tomography (CT) scan of the chest/ abdomen/pelvis showed: 1) moderate mediastinal adenopathy; 2) soft tissue mass growing into the pericardium anteriorly; and 3) large bilateral pleural effusions (Fig. 3).

The following day, he underwent right thoracotomy with placement of two epicardial leads along with mediastinal mass biopsy and bilateral chest tubes placement. Pathology results showed DLBCL with high levels of BCL2 and C-MYC. Immunohistochemical staining showed: positive cluster of differentiation (CD)20, BCL6, MUM1 and P53. Fluorescence in situ hybridization (FISH) studies showed: negative doubleHIT for DLBCL. Biopsies from the pericardium and xiphoid bone showed no signs of invasion from the B-cell lymphoma. 


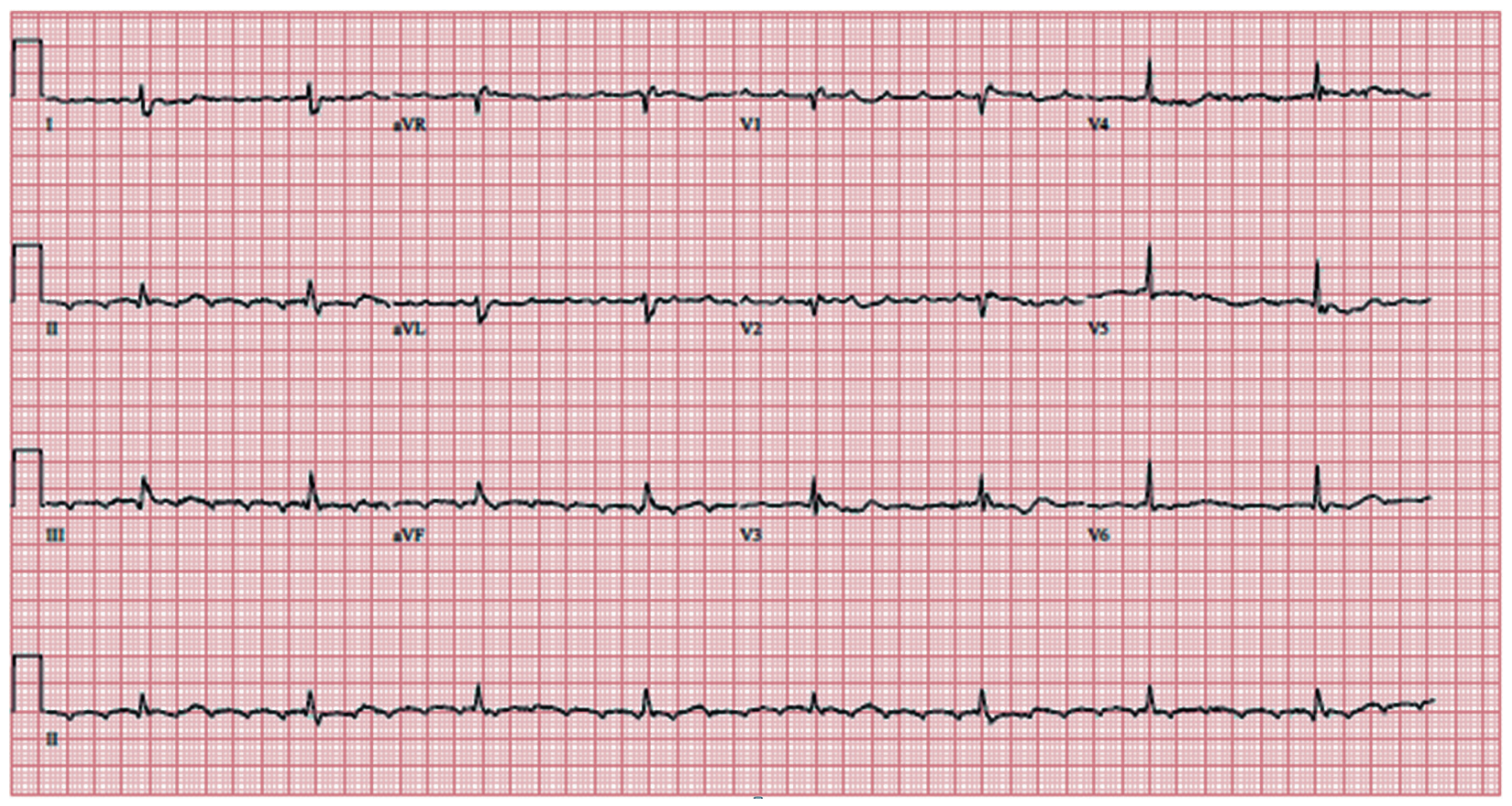

Figure 1. Atrial flutter with 4:1 block.

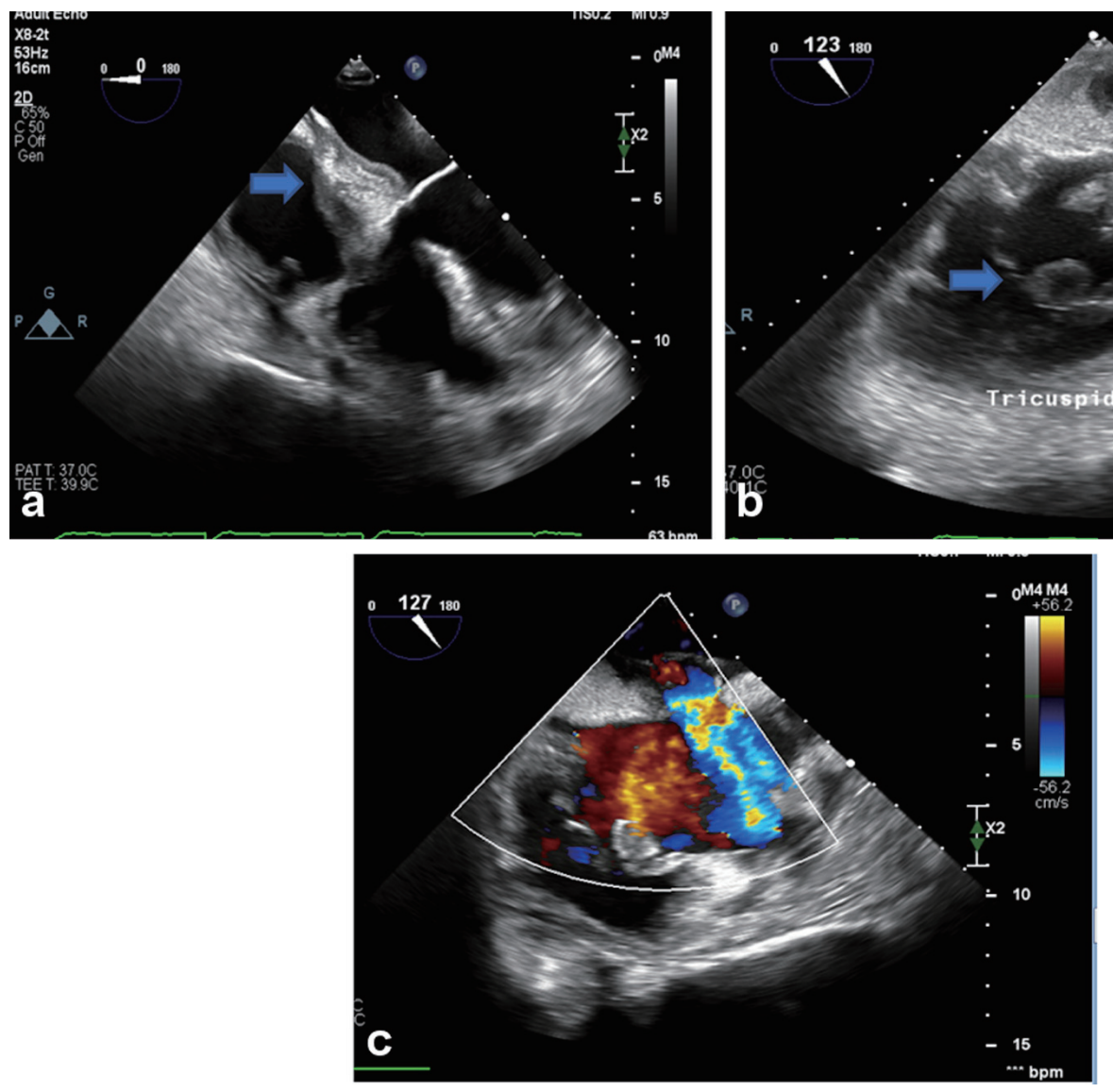

Figure 2. (a) Interatrial septal wall thickening. (b) Tricuspid valve mass. (c) Interatrial septal wall perforation and tumor expansion to left atrium. 


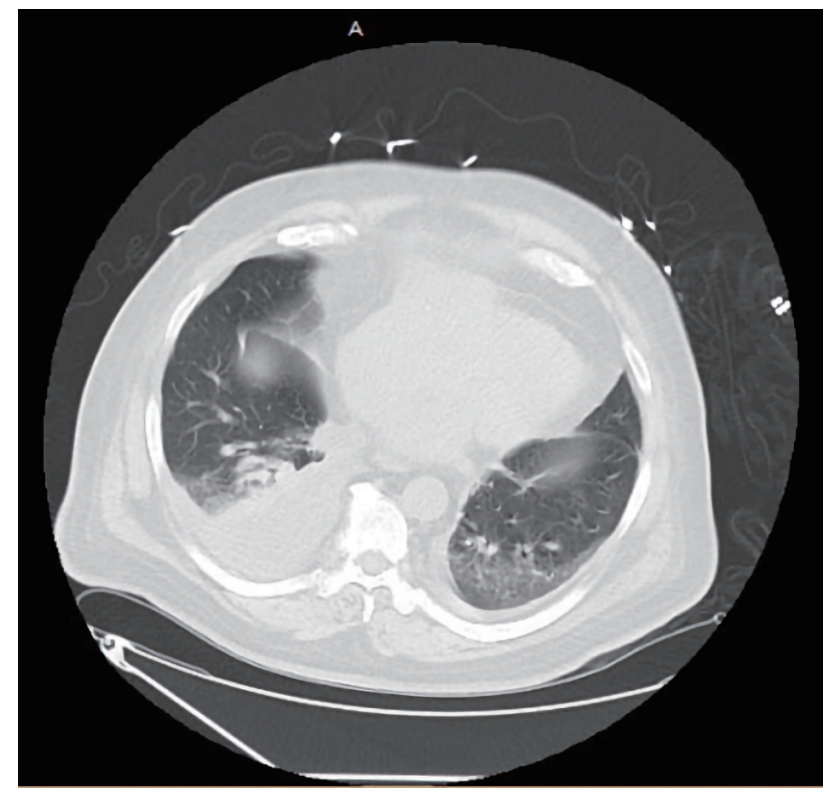

Figure 3. CT scan chest: right-sided pleural effusion, anterior mediastinal mass. CT: computed tomography.

Pleural fluid cytology was negative for malignant cells.

\section{Treatment}

Afterward, the patient was started on chemotherapy with rituxan-cyclophosphamide, doxorubicin, vincristine, prednisone (CHOP), pulse steroids and rasburicase for tumor lysis syndrome prevention. His hospital course was complicated with worsening kidney function and septic shock, managed by dialysis and broad-spectrum antibiotics including vancomycin $1 \mathrm{~g}$ intravenous (IV) q12h and ertapenem $15 \mathrm{mg} / \mathrm{kg} / \mathrm{dose}$ IV. Patient required multiple blood and platelets transfusion while on chemotherapy. Given his unresolving, recurrent bloody pleural effusions, he underwent right-sided video-assisted thoracoscopic surgery (VATS) and decortication.

\section{Follow-up and outcomes}

After receiving three cycles of R-CHOP chemotherapies, patient had improved gradually, kidney function improved, and dialysis was stopped. A follow-up TEE showed: 1) regression of tricuspid valve mass and the interatrial septal wall thickness; 2) regression of the interatrial septal tumor thickness (Fig. 4).

\section{Discussion}

Primary cardiac lymphoma primarily involves the heart, pericardium, or both [1]. While most cardiac malignancies are sarcomas [1], primary cardiac lymphoma is a rare disease, constituting about $0.5 \%$ of all extra nodal lymphomas and around $1.3 \%$ of all cardiac tumors [2]. Worldwide, the highest num-

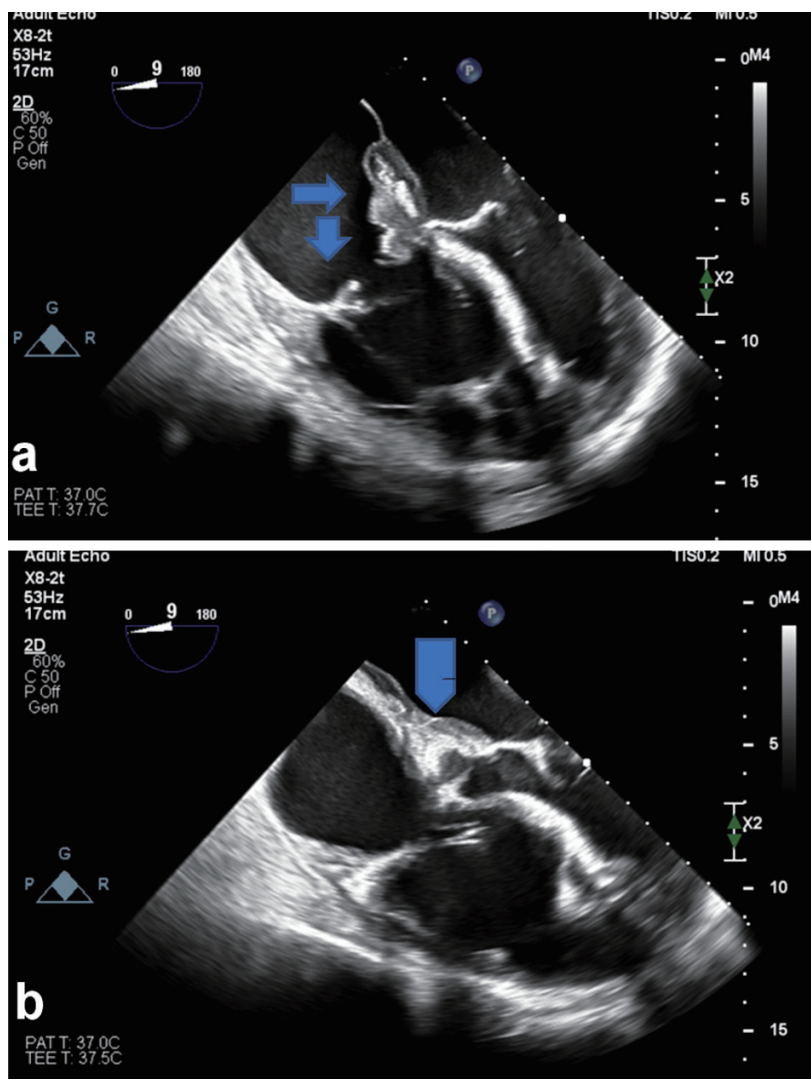

Figure 4. (a) Follow-up TEE shows regression of tricuspid valve mass and the interatrial septal wall thickness. (b) Regression of the interatrial septal wall tumor thickness. TEE: transesophageal echocardiogram.

bers of cases are seen in Asia followed by Europe, Japan and USA [2]. Usually, the cases are commonly seen in the elderly females with median age of 64 [3]. Although it is mostly seen in immunocompromised patients such as patients with acute respiratory distress syndrome (ARDS), transplant recipients or patients on immunosuppressant drugs [3], our case was an immunocompetent male with a negative HIV test.

Among the different subtypes, DLBCL is the most common [2]. Commonly the lymphoma involves the right side of the heart (right atrium, right ventricle) but can also involve the major vessels like aorta, pulmonary artery, superior vena cava (SVC), inferior vena cava and even the valves [2]. Our patient was found to have a DLBCL on the right side of the heart with interatrial septal wall perforation and left atrial expansion. Petrich et al hypothesized a possible theory to explain the more common involvement of the right side, mentioning that the thoracic duct drains into the SVC and into the right side of the heart exposing it to an already pre-exposed nodal lymphoma [4].

Patients most commonly present with symptoms of dyspnea, chest pain, syncope, but they can also present with different types of arrhythmias such as atrioventricular block, atrial fibrillation, supraventricular tachycardia, right bundle branch block, sinus bradycardia, ventricular tachycardia and rarely ventricular fibrillation which can lead to sudden cardiac arrest [2]. It can also be associated with constitutional symptoms 
such as fever, chills, night sweats and weight loss [5]. Also, if the cardiac mass is compressing the coronary arteries, the patient may present with a myocardial infarction [2].

Given the nonspecific symptoms at the time of presentation, diagnosis can be challenging [6]. Arrhythmias are usually explained by the infiltration of the myocardium and the conduction system by the lymphoma cells [1]. Also, the involvement of the right atrium leads to increased chances of interference with the sinus atrial lymph node causing arrhythmias as well [4].

Different imaging modalities are available including echocardiogram, CT scan and magnetic resonance imaging (MRI). Biopsies and histopathological evaluations are required to confirm diagnosis [7].

Regarding the treatment, chemotherapy has shown to have the greatest effect on survival [4]. A study compared CHOP therapy with $\mathrm{CHOP}$ plus rituximab in patients with newly diagnosed DLBCL especially elderly, higher survival rate was associated with rituximab with CHOP therapy had higher event free survival $(\mathrm{P}<0.001)$ and overall survival $(\mathrm{P}=0.007)$ without significant increase in drug toxicity [8]. Another study showed the increasing use of rituximab in comparison with radiotherapy [2]. Surgery can be helpful to relieve mass obstruction effects, but unlikely to alter the overall prognosis [5]. Further studies showed that surgery and radiation have no impact on survival [4].

Given the high successful rate of treatment and improved survival, follow-up closely for any relapse is important [9]. For instance, central nervous system (CNS) relapse after complete remission was reported [9]. Follow-up echocardiogram is an important way to follow up for any chemotherapy-induced cardiovascular toxicity [10].

A survival study analysis showed a median progression free survival (PFS) of 24 months in patients with primary cardiac lymphoma with 59\% complete response rate, and overall response rate of 79\%. It also showed that patients with arrhythmia had a shorter PFS compared to patients without arrhythmia (hazard ratio (HR): $0.334,95 \%$ confidence interval (CI): $0.112-0.999, \mathrm{P} \leq 0.05)[2]$.

Petrich et al reviewed 197 cases of PCL through 1949 to 2009, showing median overall survival of 3.5 months for immunocompromised patients (HR: 0.29, 95\% CI: $0.13-0.68$, $\mathrm{P}=0.004$ ); while they were not able to determine for immunocompetent patients. Interestingly it showed improved survival in patients with arrhythmia than those without arrhythmia in contrast [4]. Also, Petrich et al proposed that patients with arrhythmia presented to the hospital earlier because of symptoms due to arrhythmia and underwent earlier imaging with treatment, which can explain the improved survival in that subgroup.

\section{Conclusions}

Early diagnosis and treatment of DLBCL with appropriate chemotherapy regimen will have a significant impact on patient's survival. Our case will increase the awareness of the prevalence of primary cardiac lymphoma in immunocompetent patient populations.

\section{Acknowledgments}

We are grateful to the Department of Cardiology at Jersey Shore University Medical Center for their cooperation in this study.

\section{Financial Disclosure}

This project was not supported by any grant or funding agencies.

\section{Conflict of Interest}

The authors declare that there is no conflict of interest regarding the publication of this paper.

\section{Informed Consent}

Not applicable.

\section{Author Contributions}

Each author has individually been involved in and has made substantial contributions to conception and design, acquisition of data, analysis, interpretation of data, drafting and editing the manuscript.

\section{Data Availability}

The authors declare that data supporting the findings of this study are available within the article.

\section{References}

1. Dellas C, Chapuy B, Schweyer S, Hasenfuss G, Hunlich M. A rare cause of sudden cardiac arrest: primary cardiac lymphoma. Clin Res Cardiol. 2009;98(8):509-511.

2. Chen H, Qian S, Shi P, Liu L, Yang F. A presentation, treatment, and survival analysis of primary cardiac lymphoma cases reported from 2009 to 2019. Int J Hematol. 2020;112(1):65-73.

3. Chen KW, Chang JH, Yeh SP, Lu CR. Primary cardiac B-cell lymphoma with atrioventricular block and paroxysmal ventricular tachycardia. J Cardiothorac Surg. 2012;7:70.

4. Petrich A, Cho SI, Billett H. Primary cardiac lymphoma: an analysis of presentation, treatment, and outcome patterns. Cancer. 2011;117(3):581-589.

5. Jonavicius K, Salcius K, Meskauskas R, Valeviciene N, Tarutis V, Sirvydis V. Primary cardiac lymphoma: two cases and a review of literature. J Cardiothorac Surg. 
2015;10:138.

6. Zhong L, Yang S, Lei K, Jia Y. Primary cardiac lymphoma: A case report and review of the literature. ChineseGerman Journal of Clinical Oncology. 2013;12(1):43-45.

7. Johri A, Baetz T, Isotalo PA, Nolan RL, Sanfilippo AJ, Ropchan G. Primary cardiac diffuse large B cell lymphoma presenting with superior vena cava syndrome. Can J Cardiol. 2009;25(6):e210-212.

8. Coiffier B, Lepage E, Briere J, Herbrecht R, Tilly H, Bouabdallah R, Morel P, et al. CHOP chemotherapy plus rituximab compared with CHOP alone in elderly patients with diffuse large-B-cell lymphoma. N Engl J Med.
2002;346(4):235-242.

9. Jung YH, Woo IS, Ko YJ, Lee JH, Lim JW, Han CW. A case of primary cardiac lymphoma showing isolated central nervous system relapse. Clin Lymphoma Myeloma Leuk. 2014;14(1):e31-33.

10. Linschoten M, Kamphuis JAM, van Rhenen A, Bosman LP, Cramer MJ, Doevendans PA, Teske AJ, et al. Cardiovascular adverse events in patients with non-Hodgkin lymphoma treated with first-line cyclophosphamide, doxorubicin, vincristine, and prednisone (CHOP) or CHOP with rituximab (R-CHOP): a systematic review and metaanalysis. Lancet Haematol. 2020;7(4):e295-e308. 